\title{
Play for Real(ism) - Using Games to Predict Human-AI interactions in the Real World
}

ROTEM D. GUTTMAN, Software Engineering Institute, CERT Division, Carnegie Mellon University, USA

JESSICA HAMMER, Human-Computer Interaction Institute, Carnegie Mellon University, USA

ERIK HARPSTEAD, Human-Computer Interaction Institute, Carnegie Mellon University , USA

CAROL J. SMITH, Software Engineering Institute, AI Division, Carnegie Mellon University, USA

\begin{abstract}
AI-enabled decision support systems have repeatedly failed in real world applications despite the underlying model operating as designed. Often this was because the system was used in an unexpected manner. Our goal is to enable better prediction of how systems will be used prior to their implementation as well as to improve existing designs, by taking human behavior into account. There are several challenges to collecting such data. Not having access to an existing prediction engine requires the simulation of such a system's behavior. This simulation must include not just the behavior of the underlying model but also the context in which the decision will be made in the real world. Additionally, collecting statistically valid samples requires that test subjects make repeated choices under slightly varied conditions. Unfortunately, in such repetitious conditions fatigue can quickly set in. Games provide us the ability to address both of these challenges by providing both systems context and narrative context. Systems context can be used to convey some or all of the information the player needs to make a decision in the game environment itself, which can help avoid the onset of fatigue. Narrative context can provide a broader environment within which the simulated system operates, adding a sense of progress, showing the effect of decisions, adding perceived social norms, and setting incentives and stakes. This broader environment can further prevent player fatigue while replicating many of the external factors that might affect choices in the real world.

In this paper we describe the design of the Human-AI Decision Evaluation System (HADES), a test harness capable of interfacing with a game environment, simulating the behavior of an AI-enabled decision support system, and collecting the results of human decision making based upon such a system's predictions. Additionally, we present an analysis of data collected by HADES while interfaced with a visual novel game focused on software cyber-risk assessment.
\end{abstract}

CCS CONCEPTS • Human-centered computing • Human computer interaction (HCI) • HCI design and evaluation methods

Additional Keywords and Phrases: AI interfaces, Testing, Games, Decision support systems

ACM Reference format:

Rotem Guttman, Jessica Hammer, and Erik Harpstead 2019. Play for Real(ism) - Using Games to Predict Human-AI interactions in the Real World. In Proceedings of the ACM on Human-Computer Interaction, Vol. 5, CHI PLAY, Article 228 (September 2021), 17 pages, https://doi.org/10.1145/3474655

Permission to make digital or hard copies of all or part of this work for personal or classroom use is granted without fee provided that copies are not made or distributed for profit or commercial advantage and that copies bear this notice and the full citation on the first page. Copyrights for components of this work owned by others than ACM must be honored. Abstracting with credit is permitted. To copy otherwise, or republish, to post on servers or to redistribute to lists, requires prior specific permission and/or a fee. Request permissions from Permissions@acm.org.

Copyright ๔ ACM 2021 2573-0142/2021/September - Art228 \$15.00

https://doi.org/10.1145/3474655

PACM on Human-Computer Interaction, Vol. 5, No. CHI PLAY, Article 228, Publication date: September 2021. 


\section{INTRODUCTION}

Cyber experts have years of accumulated experience studying and counteracting the role of humans in attacks against traditional software systems. A combination of design, procedures, and training are necessary to mitigate the dangers posed by phishing, business email compromise, social engineering, and similar vectors. Technology is only as secure as its weakest points, and humans are susceptible to being over-trusting of technology and people, feeling pressure to perform, and more. In addition, the field of AI is still in its infancy and the new vulnerabilities introduced by human-AI interactions are not well understood, making them an important factor to consider in ongoing security design.

Recent misuses of systems utilizing AI highlight this point. In Flint, Michigan a useful ( $>80 \%$ accuracy) lead water line prediction engine was temporarily abandoned in favor of a legacy solution ( $<20 \%$ accuracy) (Madrigal, 2019). The Center for Privacy and Technology at Georgetown recently released a report detailing many misuses of facial recognition software by police departments, including a case where a detective received no matches on a suspect's photo and input a celebrity photo as a substitute (Garvie, 2019). In both cases, the AI system was operating as designed, but the way it was (or was not) utilized created the failure.

In order to best understand the potential (mis)use of AI-enabled systems, we must collect data on how these systems will be used under a variety of circumstances. The situations users find themselves in, and the (at times perverse) incentives associated with these situations, lead to actions like substituting a suspect's photograph with a celebrity's in order to ensure a result is found. Once we have data on how these systems will be used, we can make informed predictions on when AI-enabled systems may be misused, and how best to mitigate the chances that systems being misused will cause harm.

The current research literature is insufficient to provide actionable guidance to designers of AI decision support systems. While there are distinct bodies of research literature relevant to this problem of human-AI interaction, there is, as of yet, very little work integrating research from different disciplines. One such discipline is psychology, specifically research focused on human decision-making and how humans interpret and react to uncertainty. The second body of research comes from nascent research into Explainable AI.

The Human-AI Decision Evaluation System (HADES) described in this paper aims to bridge these disparate research communities by providing a low-friction capability for researchers to collect actionable data on real users' behavior when interacting with any proposed AI system. A key capability of this system is that it does not require the researchers to actually implement the AI system in order to evaluate it, but if an implemented system is available it can be natively "slotted-in" for testing. This slot-in capability allows for the data driven design of a system prior to implementation as well as testing the resultant system's performance to ensure it meets the design requirements.

\section{RELATED WORK}

Many types of system failures can be caused by human-AI interfaces. In this paper, we explore poor decisions based on overriding an AI-enabled decision support system. Choosing a poorlyperforming legacy system over a useful prediction engine, and manipulating the system with false data to accomplish a pre-determined goal, are both examples of humans overriding the AI to produce a worse outcome. 


\subsection{Framing and decision-making}

One way to understand system failures caused by human-AI interfaces is to consider them an extension of existing failures of human decision-making. The interface presents data on the AI's recommendation or behavior, and the human ignores or overrides that data. To better understand this behavior, we examine how people misunderstand decision-making data in other contexts.

Framing, or the way in which information is presented, is known to affect decision-making. The same information, presented in different ways, can lead to different conclusions. In a classic early study, Kahneman and Tversky show that presenting information about a medical treatment with a positive framing (how many lives it would save) led to more people choosing it (1981). The framing effect has been shown to generalize to other domains such as privacy, where presenting what a privacy policy prohibits changes user behavior compared to presenting what it allows (Samat \& Acquisti, 2017).

Framing effects are sensitive to the context in which they are presented. Kühberger showed that across 136 empirical papers that reported framing experiments with nearly 30,000 participants in all, effect sizes were considerably influenced by procedural features of experimental settings (1998). Druckman demonstrates that giving participants in framing experiments access to credible advice reduced or even eliminated framing effects (2001). Context can also provide social norms that affect participants' choices. Day et. al. examine these norms in a classroom cheating context (2011). While only one aspect of student personality (conscientiousness) is predictive of the likelihood of cheating, multiple aspects of the classroom setting (culture and pedagogy) are predictive of cheating behavior.

Taken together, these studies suggest two things. First, the way information is presented by the decision support system may have an influence on the resultant human decision. Second, the context in which the information is presented both moderates the framing effect size, and can introduce its own predictive effects based on social norms. To better understand the way that AI system information can be presented to humans, we turn to the field of explainable AI (XAI). To explore how context can be communicated in a research context, we look at the case of games.

\subsection{XAI and communicating system rationale}

Humans making decisions with AI-enabled decision support systems are likely to approach the system with their preexisting mental models of the work. Understanding those mental models will inform appropriate design of AI systems - providing an interaction that matches the users' comprehension, how they want to interact with the system, and when they may need explanations (Villareale \& Zhu, 2021). The study of XAI has been in development since the 1970's and generally refers to two different types of experiences:

- Humans developing and maintaining AI systems that need to have a detailed understanding of the models

- Humans interacting with the system as end-users need clear and relevant explanations to enable them to "understand, appropriately trust, and effectively manage... artificially intelligent partners" (Turek, 2019).

For this work we are focusing on the latter, and exploring how (or if) XAI supports end-users' understanding of why (sources, confidence, etc.) the AI system has made recommendations; and if the information provided for explainability purposes was supportive in their decision making. In their 2018 paper Narayanan et-al showed that output meeting the DARPA XAI program targets may not be understandable to a significant portion of the adult population $(>30 \%)$, to the extent that they could not even tell if the provided explanation is consistent with the resulting recommendation. 
The XAI approach supports effective human use of AI systems by providing explanatory information for the primary users, using language they understand, and in-context of the purposes of the system. This approach requires the system be designed using user-centered practices and the people creating the system be aware of cognitive biases that affect human interactions with systems and each other (Miller, Howe \& Sonenberg, 2017). Autonomy bias and anchoring effects are common issues that need to be managed via design and Yin et. al. show how the initially claimed accuracy of an AI-enabled system can influence a human's ongoing trust in that system (2019). While Yin et. al. do not explicitly link their findings to the cognitive bias literature, other researchers are working to fill the gap between decision research and XAI. Most notably, Wang et. al. developed a framework for XAI in medical contexts that addresses the representativeness, anchoring, availability, and confirmation biases (2019). They demonstrate how countering each type of bias can drive specific design decisions in XAI systems, and validate their work through co-design in a medical context.

AI systems do not exist in a vacuum, and teams need to consider how the system will be compared to existing parallel experiences by the participants. For example, how does the AI system behave differently as compared to other systems/human interactions and are there signals expected in these interactions that are absent because it is an AI system (Gero et-al, 2020)? Interestingly, some prior research suggests that the perceived source of system information may also matter in terms of framing and decision-making. For example, Geiskovitch et-al showed that humans are willing to continue a task despite voicing a desire to stop for much longer if instructed to do so by a robot (rather than a human). Conversely, Okita et. al. showed that participants learned more from a computer system if they believed it was being operated live by a human, compared to believing it was an autonomous agent (2007). Questions such as these can be studied by embedding XAI content into the context of a game.

\subsection{Games as experimental testbeds}

Games are commonly used as interventions in decision-making research. Sometimes, they are "games" in the sense of game theory (Dufwenberg, Gachter \& Henning-Schmidt, 2006), but sometimes fully-featured play experiences are used (e.g., Rumeser \& Emsley, 2019). In the latter case, there are two key features that games provide.

First, games provide systems context for decisions. When the player makes a choice in the game, they gather information from the game's environment about the likely consequences. Information might include rapidly-processed cues such as how fast an enemy character is approaching, but it can also include analyzing written material (Steinkuehler, 2004) or making statistical judgments (Steinkuehler \& Duncan, 2008). Players use feedback from the game (e.g., about their performance) to make hypotheses about the game's underlying system. Because game systems are typically obscured from direct inspection by the player, this situation is parallel to that of XAI.

Second, games provide narrative context for decisions. As suggested above, studying framing effects in the absence of context may provide misleading information. People adjust their decisions based on perceived social norms, as well as based on the incentives at stake (e.g., a detective being rewarded for closing cases). Games can create local sets of social norms (e.g., Bergstrom, 2010), and players typically care about the outcome of the games they play (Juul, 2010). Games can, therefore, allow us to study how framing affects human-AI interfaces in more realistic ways than laboratory experiments alone.

Both systems and narrative context can provide meaningful incentives for players. For example, a player who masters game systems can better predict whether their actions will be effective at achieving their goals. One can see this dynamic at play in games like OverCooked, 
where missing an ingredient in a dish can cause the player to lose the game. On the other hand, narrative engagement can shape players' emotional experience of the game (Journet, 2007, Schrier, 2019). In visual novels such as Doki Doki Literature Club, deciphering clues to better understand the characters is critical to solving the game's puzzles. Finally, it is important to note that systems and narratives are not cleanly separated. For example, Bopp et. al. (2018) show that players found moments of tension between systemic and narrative incentives to be emotionally challenging, such as a player of Mass Effect 1 choosing whether to save the more powerful character, or the one they liked better.

To date, games have been used in two ways related to XAI. First, games have been used as testbeds for XAI. Examples include explaining NPC behavior (Molineaux, Dannenhauer \& Aha, 2018), moderation of game communities (Kou \& Gui, 2020), and generating dialogue models (Madumal et. al., 2019). A smaller body of work explores games as ways to understand and improve AI systems' explainability. Feng and Boyd-Graber use a game to produce human analyses of AI-generated natural language (2019), while Fulton et. al. present a game prototype that can be used to study how humans select and interpret explanations for image-based deep learning algorithms (2020). However, games have not yet been used to explore framing and data presentation effects of AI system designs. We aim to fill that gap. We, therefore, present a system that allows researchers to study the effects of system information and narrative context on humanAI decision support systems within the context of a game.

\section{SYSTEM AND GAME DESIGN}

In the ideal use-case we would utilize HADES to collect data on subjects repeatedly making the same decision, while slightly modifying the specifics of the decision, in order to determine the overall quality of decisions made with a specific AI system output. Unfortunately, presented as such, fatigue quickly sets in to what becomes a monotonous series of tasks. Thankfully, repeatedly making similar decisions is a hallmark of many types of gameplay. While the particular context and variables associated with a decision may change, the core game mechanic often remains the same from turn to turn or scene to scene. For example, the core game mechanic of Monopoly is the choice of purchasing a property or not. The cost of the property, its location, and what other properties on that street are owned and by whom may change from turn to turn, the decision itself does not. By designing games in which making the AI-assisted decision is the core game mechanic, we can alleviate the challenges associated with collecting useful human decision data.

\subsection{HADES API}

Previous efforts to investigate the effect of explainability on users, such as Lai and Tan's system for detecting deception (2019), and Ribeiro et-al's system for choosing applicable classifiers (2016) must have included the capability to provide AI decision support system predictions to users and to collect feedback about the resultant user behaviors. However, without a publicly available system for providing the tasks and associated predictions, each group of researchers is forced to re-invent the wheel in order to conduct their research. As such, we designed the HADES API to alleviate this duplication of efforts by segmenting the challenge of providing AI decision support system outputs and storing resultant observations of user behavior from the challenge of designing the system in which the users will be operating. The HADES API serves as a generalizable engine, which can be easily integrated with any user facing systems.

This generalizability allows HADES to drive either prototype systems designed specifically for an experiment or the front-end of a production system, without significant modification. In fact, we believe the HADES API could support any experimental design within the application 
grounded evaluation or human grounded metrics categories of the Doshi-Velez \& Kim Taxonomy of Interpretability Evaluation (2017).

In order to allow our system to collect data from as wide a variety of sources as possible, we engineered HADES to expose a RESTful API that enables both the distribution of task data and AI predictions associated with each task, as well as the collection of data about the human's decision making. At a high level, the functionality of the HADES API can be broken down into two categories: Task functionality and User functionality. In this section we will describe the use of the HADES API in the context of a game, though HADES API consumers can be any software application, including deployed AI decision support systems.

HADES Task API endpoints allow a game to request a task or set of tasks from the API. The returned task object contains all the information needed for a human to make their decision. In the Monopoly example, the Task would contain the name of the property, the cost of the property, ownership information about other properties on the same street, and so forth. The specific items contained in a task object are configurable based upon the game's data requirements. Depending on the specific game's needs, all tasks can be requested at the start to allow for initialization, or requests can be made iteratively throughout the game on an as-needed basis.

HADES User API endpoints are designed to enable the management of all aspects of an individual game's user's interaction with the test harness. The first call made to the User API will create the user and assign them a randomly generated user identifier (GUID) in order to allow for their behavior throughout the game to be tracked while maintaining their anonymity. At the same time, the HADES API will assign this user to an experimental condition. The experimental condition can be randomly assigned, preconfigured, or determined based on existing data criteria (such as ensuring a set of experimental conditions have a specified number of participants before assigning participants to a secondary set of experimental conditions).

Additional HADES User API endpoints allow the game to request AI predictions for any task. Note, these predictions need not be the output of an actual AI system. HADES allows researchers to pre-seed predictions and select from them in such a manner as to mimic the desired statistical behavior of a proposed AI system. If an actual AI system is available, HADES allows the AI system to be slotted in and to provide its own recommendations. This choice of data source is transparent to the game so as to allow for native testing of actual AI systems without any reconfiguration.

Finally, the HADES User API provides an endpoint for the collection of data about a user's decision making. The minimal data required is the outcome of their decision, however additional data collection is configurable if desired. This additional data collection can include information such as tracking what information the user reviewed or ignored and for how long, information regarding what interactions with the system the user engaged in, or even full logging of every click the user made within the game. The only limitation is based on the data storage allocated to the HADES API and the bandwidth available for communicating between the game and the API endpoint.

HADES was made available to several game design groups at the Carnegie Mellon University Entertainment Technology Center. These groups created multiple novel game experiences utilizing the HADES API, and one was selected for continued use in this program. Ultimately, the selected program was chosen because it integrated the decision-making process intrinsically into the gameplay (Habgood \& Ainsworth, 2011, Aleven et. al., 2010), whereas other candidates provided games that included the decision-making process as a separate element of the game. 


\subsection{Game Design Criteria}

The primary goal of our experiment was to be able to examine the effects of a variety of AI decision support system designs on both human trust (via proxy) in the AI system, as well as on the quality of the resultant decision making. Our set of candidate design elements was broadly divided into two categories - explainability elements and contextual elements. Explainability elements relate to providing information on why the AI system made the recommendation that it gave, while contextual elements affect the context within which the decision is made (risks and rewards). In game terms, these map to system feedback and narrative elements.

In order to evaluate the explainability elements, our design had to feature a modular capability for expressing the AI's recommendations as their content could drastically vary depending on the assigned experimental condition. In the most extreme cases where all explainability elements are disabled the user might receive a single word recommendation as to which choice to pick, whereas at the other extreme they might receive all of the following:

1. The recommendation

2. A confidence value for that recommendation

3. A list of the input information which the AI utilized to make this decision (windows API calls made by the software, antivirus scan results, signer information, etc.)

4. Highlights within the input information identifying which specific elements the AI relied on most heavily to make its recommendation.

To evaluate the impact of narrative contextual elements, our design had to support multiple narratives that could vary the level of endogenous risk to the player character, while retaining the same core gameplay and decision points across all narratives. Further, it was important for the tasks to be motivated by the game's story in order to imbue them with purpose, rather than for them to be disconnected prompts. This kind of integration between task, mechanics, and narrative has shown benefits for user engagement and learning across a number of serious game contexts (Habgood \& Ainsworth, 2011; Laine \& Prestopnik, 2017; Habgood, Ainsworth \& Benford, 2005).

Finally, we wanted to recognize that sometimes players are simply uncertain. However, we did not want to let players effectively opt out of the study by indicating uncertainty in response to every choice. To address this, we decided to allow players a limited number of opportunities to forward the request to a subject matter expert. This allows the player to express their lack of confidence in their ability to interpret the problem while still heavily encouraging them to make a decision themselves where possible.

\subsection{TGI Launch Day}

TGI Launch Day is a visual novel game designed to integrate with HADES to test how both contextual and design factors impact users' reactions to an AI recommender system. In the game, players take on the role of a security analyst at a software company and are tasked with fielding user requests for approval to run different software on company computers. Players are assisted in this task by an AI system that analyzes the requested software and provides a recommendation for whether the software should be approved or not. To support the HADES system the game supported a number of design variations, which we explore in this study. 


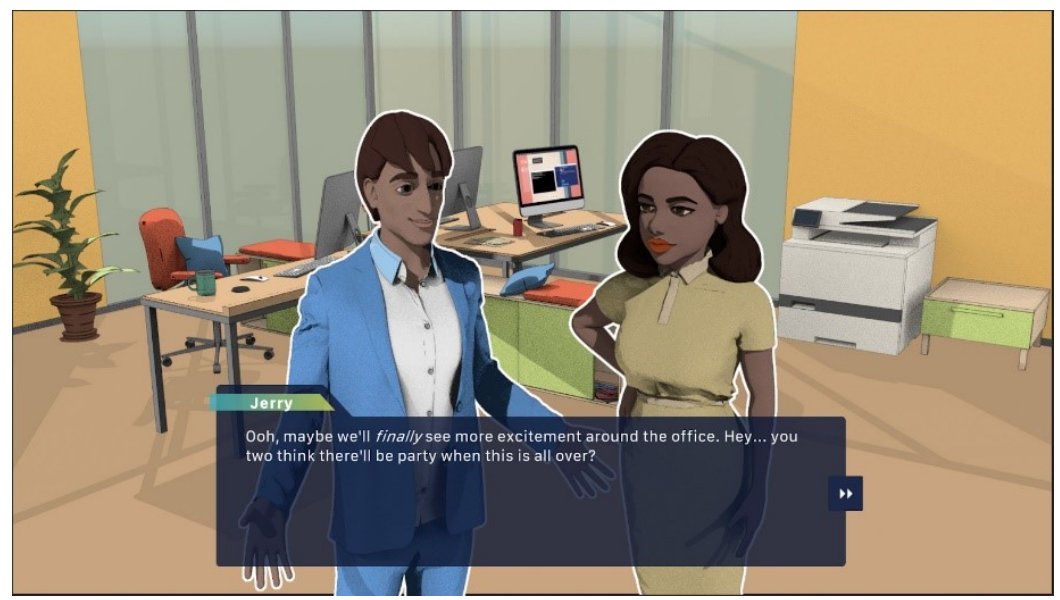

Figure 1. A screenshot from a narrative scene in the game

During the visual novel portions of the game, players engage in scripted dialogue sequences with in-game characters (Figure 1). One design variation manipulates the narrative stakes of the story that takes place during these dialog sequences. In the low-stakes version of the story, the player and their colleagues have heard rumors of a launch party for their company's new product to which they will get invited if they perform well in their job. In the high-stakes version, a malicious hacker is targeting the company and the player must remain extra vigilant or else a critical product launch may be compromised. These high- and low-stakes conditions were iteratively developed during the playtest process. For example, in direct comparisons between the two conditions, players were consistently able to identify the higher-stakes version; when played independently, players reported feeling more anxious for the characters to succeed when the stakes were higher. Counterproductive elements (such as celebrities attending the low-stakes party) were identified, and either revised or removed. 


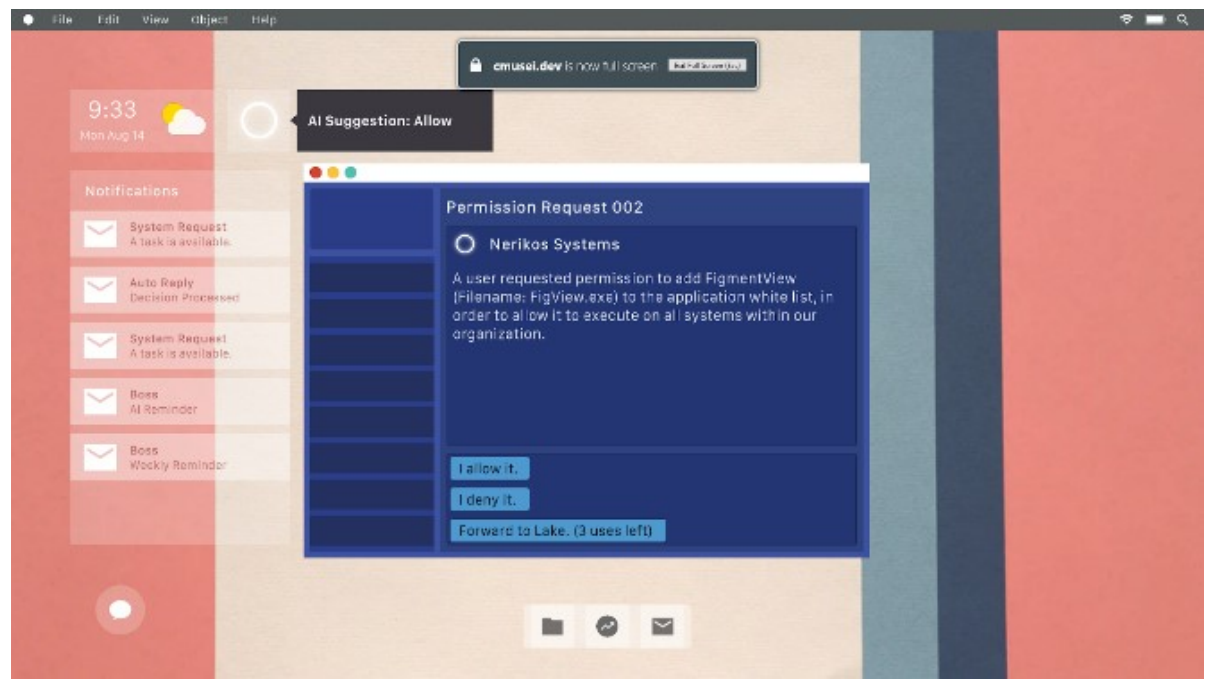

Figure 2. A screenshot of the decision task interface in the no information condition

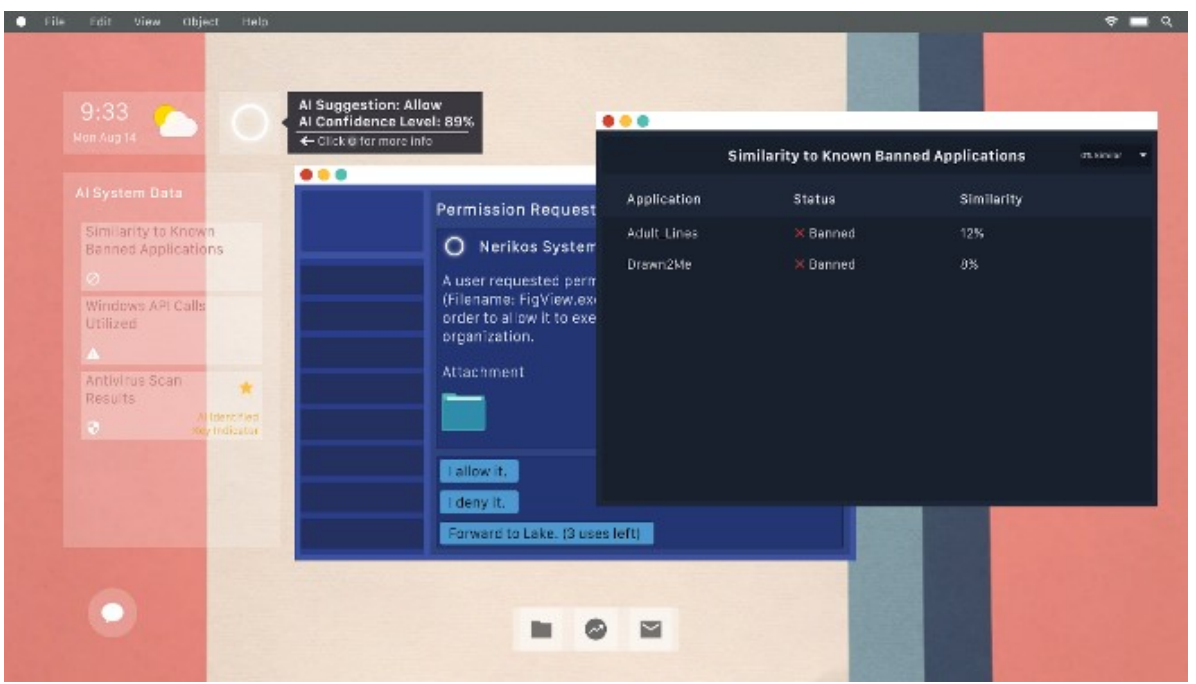

Figure 3. A screenshot of the decision task interface in the full information condition

To connect the decision tasks with the narrative, they are framed as emails sent to the player by coworkers requesting approval for various software to run on company computers. Players may choose to approve or deny requests, which are evaluated as correct or not. Alternatively, they have a limited number of opportunities to forward requests on to the security specialist (Lake) who has limited capacity to deal with issues where the player is unsure. When players forward requests to Lake, it is considered to be declining to make the decision. After the player has completed 8 of these tasks, they start to receive a performance report (Figure 4) after each task. The performance report tracks their decision correctness against the performance of the AI system so they can understand how well they are doing. If the player makes too many mistakes, there are in-game consequences such as other characters being upset with them and, eventually, the failure of the party or product launch. 


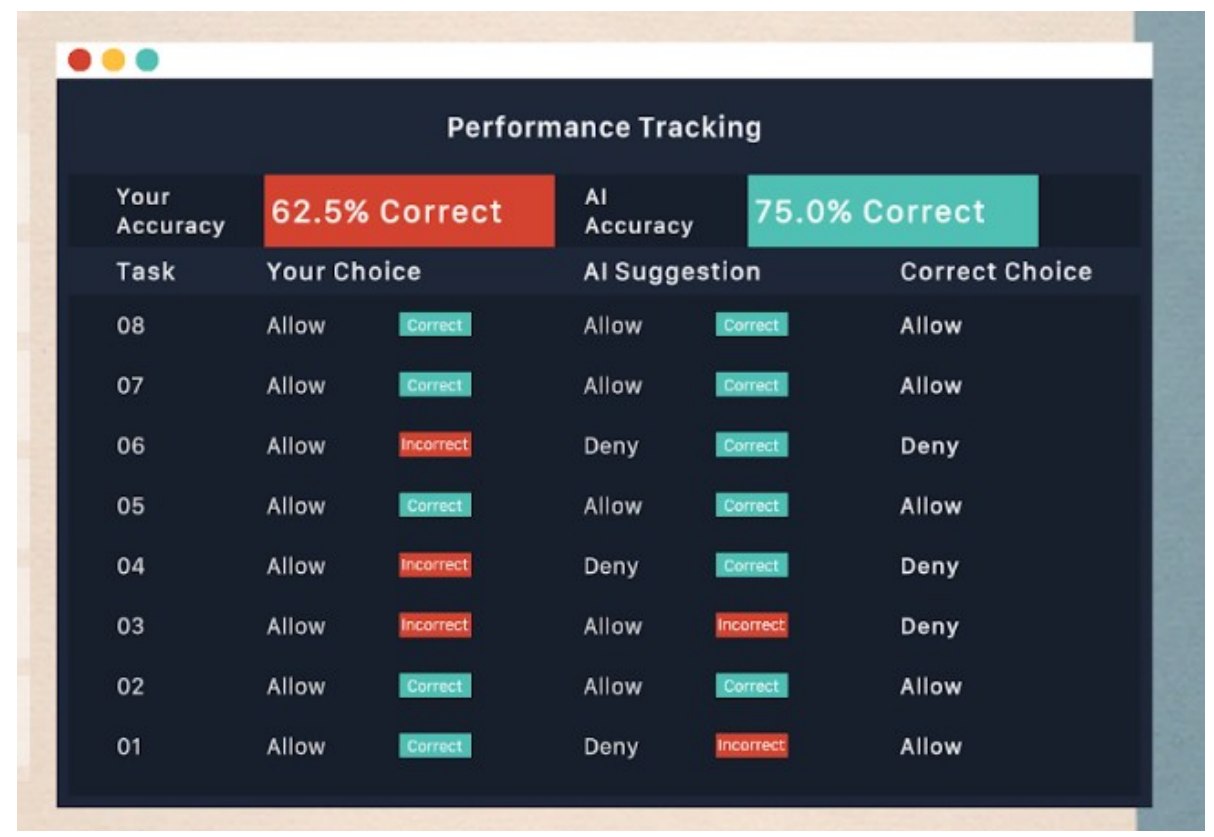

Figure 4. A player's performance report sent after each task once 8 tasks have been completed

The decision task interface is designed to support a number of condition variations that provide or withhold additional information about the AI's decision-making process to the user. In this initial study we explored the far extremes of these features with a low information condition (Figure 2), which provides no additional information to support the AI's recommendation, and a full information condition (Figure 3), which provides a number of different resources for users to consult in making their decisions. Most saliently, the full information condition provides an estimate of the AI's confidence along with its recommendation. This number is sampled within HADES based on the simulated AI's pre-defined accuracy of $85 \%$. In addition to the confidence estimate, the full information condition also provides diagnostic resources describing additional factors (such as the similarity of the request to known banned applications) the AI would have taken into account in making its recommendation.

In other words, the narrative stakes of the game can be high or low, depending on player condition. From a systems perspective, the player may have more or less information about how the AI makes decisions, and therefore about how to use the AI's recommendation as part of their own decision. The first eight items, with no feedback, provide a baseline; once the player begins receiving feedback on correctness, they can use explainability information (if present) to improve their performance on the tasks. Finally, task performance links back to the narrative by impacting how the party turns out, and whether the player wins or loses the game.

\section{METHODS}

To demonstrate the potential of the HADES system for measuring users' reactions to different AI designs, we performed a user experience study of TGI Launch Day with participants recruited through Amazon Mechanical Turk. The participant pool was limited to English speakers residing in the United States.

We feel confident the results obtained via Amazon Mechanical Turk are representative as the research community has repeatedly shown that well known studies (originally performed utilizing 
more traditional means) can be successfully replicated in MTurk (Mullinix et. al., 2015; Berinsky, Huber \& Lens, 2012; Clifford, Jewell, \& Waggoner, 2015; Weinberg, Freese, \& McElhattan, 2014). In many cases MTurk samples can yield higher quality data than student samples, community samples, or even high-quality national samples, including similar tools such as Qualtrics's qBus (Anson, 2018; Thomas \& Clifford, 2017).

However, one of the key findings of the body of research on MTurk data collection is that it is critical for the candidate pool to be carefully controlled, and the data collection method to be correctly tuned to align with MTurk. As such, we adopted several measures to ensure high quality data collection. These measures can be divided into two broad categories, Participant Filtering and Data Validation.

Participant filtering techniques prevent the collection of data from individuals unlikely to yield high-quality results. These techniques were implemented both within the MTurk platform, and within our data collection front-end. Within the MTurk platform, we configured our Human Intelligence Task (HIT) to ensure that it is only presented to respondents with a minimum previous HIT approval rate of $98 \%$ or higher, and at least 100 previous HITs completed. The total completion requirement is due to a quirk in the MTurk platform, which causes users with less than 100 completed tasks to be considered to have a $100 \%$ approval rate. Setting a HIT approval rate of $95 \%$ or higher has been shown to be sufficient to dramatically increase collected data quality (Peer, Vosgerau, \& Acquisti, 2014).

In addition, we have found source IP filtering to be of critical importance. It has been shown that the vast majority of low-quality responses to MTurk data collection efforts come from participants utilizing a VPN service, likely in order to fraudulently be considered a US person and thus qualify for US based MTurk tasks (Ahler, Roush \& Sood, 2018; Dennis, Goodson \& Pearson, 2020). We utilized VPN detection methods to block those connections originating from nonresidential IP addresses, as well as those known to host VPN service endpoints from within residential networks. This technique has been shown to be effective at preventing the vast majority of the problematic users (Kennedy et. al., 2020), as it is not worth their time to attempt to circumvent the protection techniques when they are encountered, since most HITs do not implement such protections. By implementing these protections in an automated manner as a part of the data collection front-end we were able to avoid the need for collecting the participants IP addresses to further maintain their anonymity.

In addition to the participant filtering controls available through Mechanical Turk, we implemented additional acceptance criteria for inclusion in our study data. First, we included two attention check tasks within the first 8 items in the game where players were asked to evaluate permission requests for two obvious cases (Notepad.exe to be accepted, and MALWARE.EXE to be rejected). If players responded incorrectly to either of these items they were excluded from further analyses (though they were still compensated). Second, we removed any players who completed the game too quickly. Through internal playtesting we determined a minimum of 7 minutes was required to complete the entire game while still substantively reading all the narrative text. It should be noted, the implementation of these protection techniques has not been shown to introduce bias into the candidate pool in previous research programs (Kennedy et. al., 2020).

Our study employed different compensation schemes over multiple data collection windows. The first data collection window took place from Oct 29 - Nov 4, 2019 and players were compensated $\$ 2.25$ for completing the whole task. The second data collection window took place from Dec 2 - Dec 4, 2019 and players were compensated \$1.75 for completing the task and an additional $\$ 2.00$ bonus if they accurately identified $75 \%$ of the tasks in the game. This compensation strategy allowed us to check whether performance went up when players were externally compensated for in-game performance; if so, it would inform our findings about the 
effect of in-game compensation, as it would suggest our in-game incentives for performance were insufficient.

\section{RESULTS}

Over the two sampling periods, 274 participants completed the entire game. From this initial sample we removed 129 participants for failing to correctly respond to the embedded attention check items, and an additional 4 were removed for completing the game in under 7 minutes. This left us with a final sample of 141 participants. A full breakdown of these populations by condition can be seen in Table 1. Note, the High Compensation condition was run for fewer days resulting in a lower overall number of participants.

Table 1. Number of participants in each condition

\begin{tabular}{|l|l|l|l|l|}
\hline $\begin{array}{c}\text { Information } \\
\text { Availability }\end{array}$ & Narrative Stakes & Compensation Stakes & $\begin{array}{c}\text { Number of Players } \\
\text { Completed }\end{array}$ & Final Sample \\
\hline Available & Low & Low & 47 & 22 \\
\hline Available & High & Low & 45 & 27 \\
\hline Not Available & Low & Low & 49 & 30 \\
\hline No Available & High & Low & 59 & 34 \\
\hline Available & Low & High & 19 & 11 \\
\hline Available & High & High & 23 & 6 \\
\hline Not Available & Low & High & 15 & 6 \\
\hline No Available & High & High & 17 & 5 \\
\hline
\end{tabular}

First, we wanted to verify there were no conditional effects on players' baseline performance in correctly identifying malicious software or in their baseline agreement with the AI's recommendations (a proxy for baseline trust in AI systems) on the first 8 items before they received their first feedback summary. We performed multi-way ANOVAs of each of the conditional variables on player baseline correctness and found no significant differences. Similarly, there were no significant differences in players' baseline agreement with the AI from the feature visibility or narrative stakes features, but there was a significant difference in baseline trust between compensation conditions.

To evaluate the impact of Information Availability and Narrative Stakes on players' performance identifying malicious software and AI trust we performed a repeated measures regression analysis to predict players' performance and trust in the latter portion of the game given their initial performance and trust, and conditional assignment. The results for these regression analyses can be seen in Tables 2 and 3.

Table 2: Regression Coefficients for Conditional Variables on Player Performance

\begin{tabular}{|l|l|l|l|l|}
\hline Coefficient & $\mathrm{B}$ & $\mathrm{SE}(\mathrm{B})$ & $\mathrm{p}$ & $\mathrm{S}$ ig \\
\hline (Intercept) & 0.617 & 0.029 & $<0.001$ & $* * *$ \\
\hline Time & 0.059 & 0.021 & 0.005 & $* * *$ \\
\hline High Stakes Narrative & 0.013 & 0.031 & 0.662 & \\
\hline Information Available & -0.050 & 0.035 & 0.103 & \\
\hline High Compensation & 0.021 & 0.039 & 0.589 & \\
\hline
\end{tabular}


Looking at the results for regressing condition variables on player performance in Table 2, we see a significant positive relationship for time suggesting players are generally learning to better identify malicious software as the game progresses. Looking at the conditional variables, however, we see no significant effects for the high vs low narrative stakes, the inclusion of additional AI information, or for compensating performance.

Table 3: Regression Coefficients for Conditional Variables on Player Agreement with the Al System's Recommendations.

\begin{tabular}{|l|l|l|l|l|}
\hline Coefficient & $\mathrm{B}$ & $\mathrm{SE}(\mathrm{B})$ & $\mathrm{p}$ & $\mathrm{Sig}$ \\
\hline (Intercept) & 0.726 & 0.026 & $<0.001$ & ${ }^{* * *}$ \\
\hline Time & 0.067 & 0.020 & 0.001 & ${ }^{* *}$ \\
\hline High Stakes Narrative & -0.031 & 0.029 & 0.292 & \\
\hline Information Available & -0.091 & 0.029 & 0.002 & ${ }^{* *}$ \\
\hline High Compensation & 0.081 & 0.037 & 0.028 & ${ }^{*}$ \\
\hline
\end{tabular}

Looking at the results for regressing condition variables on players' agreement with the AI system in Table 3, we see a similar significant positive relationship between time and agreement, suggesting that as the game progresses players are more likely to agree with AI system's recommendations (potentially indicating an increase in trust). Looking at the conditional variables leads to some interesting trends. Similar to the player performance metric there was no significant effect on players' agreement with the AI system from the narrative stakes. Information availability, however, shows a significant negative impact on players' agreement with the AI (see example of information availability in Figure 2). When taking both the information availability and time coefficients into account together, the combined effect suggests that players without additional information about the AI's decision-making trend toward agreeing with the AI more as the game progresses while players who have insight into the AI's decision-making stay roughly the same throughout.

\section{DISCUSSION}

Our study of TGI Launch Day provides encouraging evidence for the potential of HADES as a model for investigating users' reactions to different AI scenarios. By varying the narrative stakes, system information availability, and compensation across players we can make some conclusions about how users might react to AI recommender systems in different settings.

Across both of our outcomes, we found a general trend of participants improving over time. Our data shows participants get better at identifying malicious software requests as they play TGI Launch Day. In addition to this learning, they may also tend to trust the AI system more over time, as evidenced by the positive trend in agreement with the AI. Given that the AI system was defined within HADES to have an $85 \%$ accuracy rate at the task, it makes sense that participants would tend toward following its recommendations as they also improve in accuracy over time.

In terms of the in-game narrative condition, we observed no significant effects for either low or high stakes narratives for either of our outcome variables, even though playtest participants identified clear differences in narrative stakes. While this might suggest that the participants decision making on the software identification task is unaffected by the consequences of the choice, further research is needed to investigate whether participants appropriately understood the diegetic components of the story. One possibility for this lack of impact could be due to a 
sampling bias in the MTurk audience. If participants were engaging with the game on a voluntary, rather than compensated, basis they may value the diegetic stakes of the story more highly.

Looking at the external stakes of varying compensation, we see somewhat similar results. Interestingly, compensating participants for improved performance did not appear to lead to increased performance over and above the effect of learning the task itself. There was, however, an effect of compensation on participants' agreement with the AI system suggesting that financially motivated participants were more willing to defer to the AI's recommendation.

Finally, providing participants with additional information about the AI's reasoning resulted in interesting effects across our outcome measures. In terms of correctness there was no significant effect of information availability over and above the increase already accounted for by learning. For agreement (and by proxy, AI trust), there was a significant reversal effect of providing information to participants that counteracts the general trend of increasing trust over time. This would imply that participants who had additional insight into the AI's decision making process remained roughly as skeptical of the system as they had been initially, while participants who were not given information tended toward trusting the system in its absence.

The observed effect of explainability information on participant's agreement with the AI recommendations was counter-intuitive. Much of the current research into "Explainable AI", such as DARPA's XAI project, is focused on producing exactly this type of information with the goal of allowing users to make higher quality decisions based upon the AI's recommendations. Our results suggest that in the majority of cases, users trust in the AI recommendation will be unaffected by the presence of such explainability information. In fact, our data indicates that in the absence of confidence information, users in high stakes situations tend to increase their reliance on the AI's recommendations, yet if such users are exposed to the uncertainty in the AI's recommendations they revert to their initial baseline trust in the AI system. This may be due to autonomy bias reinforcing an incorrect assumption on the part of study participants that any recommendation given by an AI system is made with a high degree of confidence. This perceived confidence may be negated when doubt is expressed in the form of exposing the actual confidence measures, even if the stated confidence measure is higher than the performance they've come to expect from the system.

\section{FUTURE WORK}

Thanks to the flexibility of the HADES test harness and the TGI Launch Day game, there are several additional follow-up experiments that could be conducted with little to no modification of the harness. For example, the data collected during this study can serve as a useful baseline for layman attitudes toward AI decision support systems in this context. A replication of this study with a set of subject matter experts (SMEs) could be used to determine how SME attitudes differ from layman attitudes with respect to AI decision support systems.

Similarly, during the course of this study, all simulated AI decision support systems were designed to operate in a usefully informative manner (with a uniform overall accuracy of $85 \%$ ). A simple modification to this study would be to repeat the tests with a variety of simulated AI decision support systems with a variety of accuracies, from the uninformative to the infallible. Such a study could help determine the rate at which users modify their behavior under these conditions and provide useful guidance for minimal accuracies required for user-acceptance within a challenge domain.

The HADES test harness could also be used to examine user behavior in the context of adversary attacks on AI systems. The simulated AI system could be instructed to return useful information for an initial period, priming users to trust it, and then degrade its performance over 
time to simulate adversary activity. This would allow us to determine how quickly (if at all) users notice and modify their trust in the AI system based on a change in its behavior.

The flexibility of the HADES test harness should allow for a wide variety of systems to integrate with it, with minimal effort. A simple web query is all that is needed to interface with the HADES backend. This will allow other researchers to easily adopt HADES for their own research purposes. We are already exploring possible integrations with a variety of contexts (chess, math education, healthcare recommendations). The authors welcome queries from any researchers interested in utilizing the HADES system.

\section{CONCLUSION}

In this work, we present the Human-AI Decision Evaluation System (HADES), an API that allows a low-friction capability for researchers to collect data on real users' behavior when interacting with a proposed AI system. We integrated HADES with a game, TGI Launch Day, that situated a proposed AI recommendation system in a fictional company's workflow. By varying the narrative stakes of the game, the system information made available to the player, and the amount of external compensation for successful play, we were able to see how users might react to AI recommender systems in different settings. We found that participants improved at the in-game task (deciding what software to approve) over time, and that they trusted the AI recommender system more over time as well. Participants responded differently to the AI when there was a financial incentive to do so, and seemed more skeptical about the AI's results when they had more insight into how the system worked. Additionally, we demonstrated the value and flexibility of the HADES test harness, and we look forward to collaborating with other researchers who are interested in using our system.

\section{ACKNOWLEDGMENTS}

The authors wish to thank the students of the TGI Launch Day team, Mingzhi Cai, Dakota Hernandez, Angelo Pagliuca, Katherine Sabel, Natalie Schmidt, and Qiyi Tang who developed the TGI Launch Day application.

\section{REFERENCES}

[1] D. J. Ahler, C. E. Roush, and G. Sood. 2018. The micro-task market for "Lemons": Collecting data on Amazon's Mechanical Turk. Working Paper. Epub ahead of print.

[2] V. Aleven, E. Myers, M. Easterday, and A. Ogan. 2010, April. Toward a framework for the analysis and design of educational games. In 2010 third IEEE international conference on digital game and intelligent toy enhanced learning (pp. 69-76). IEEE.

[3] I. G. Anson. 2018. Taking the time? Explaining effortful participation among low-cost online survey participants. Research \& Politics, 5(3), 2053168018785483.

[4] K. Bergström. 2010, October. The implicit rules of board games: On the particulars of the lusory agreement. In Proceedings of the 14th International Academic MindTrek Conference: Envisioning Future Media Environments (pp. 8693).

[5] A. J. Berinsky, G. A. Huber, and G. S. Lenz. 2012. Evaluating online labor markets for experimental research: Amazon.com's Mechanical Turk. Political analysis, 20(3), 351-368.

[6] J. A. Bopp, K. Opwis, and E.D. Mekler. 2018. "An Odd Kind of Pleasure”: Differentiating Emotional Challenge in Digital Games. In Proceedings of the 2018 CHI Conference on Human Factors in Computing Systems (CHI '18). Association for Computing Machinery, New York, NY, USA, Paper 41, 1-12. DOI: https://doi.org/10.1145/3173574.3173615

[7] S. Clifford, R. M. Jewell, and P. D. Waggoner. 2015. Are samples drawn from Mechanical Turk valid for research on political ideology? Research \& Politics, 2(4), 2053168015622072.

[8] N.E Day, D Hudson, P.R. Dobies, et al. 2011. Student or situation? Personality and classroom context as predictors of attitudes about business school cheating. Soc Psychol Educ. 14: 261. https://doi.org/10.1007/s11218-010-9145-8

[9] S. A. Dennis, B. M. Goodson, and C. A. Pearson. 2020. Online worker fraud and evolving threats to the integrity of PACM on Human-Computer Interaction, Vol. 5, No. CHI PLAY, Article 228, Publication date: September 2021. 
MTurk data: A discussion of virtual private servers and the limitations of IP-based screening procedures. Behavioral Research in Accounting, 32(1), 119-134.

[10] F. Doshi-Velez and B. Kim. 2017. Towards A Rigorous Science of Interpretable Machine Learning. arXiv preprint arXiv: 1702.08608

[11] J. N. Druckman. 2001. Using credible advice to overcome framing effects. Fournal of Law, Economics, and Organization, 17(1), 62-82.

[12] M. Dufwenberg, S. Gächter, and H. Henning-Schmidt. 2006. The framing of games and the psychology of strategic choice (No. 19/2006). Bonn Econ Discussion Papers.

[13] S. Feng and J. Boyd-Graber. 2019, March. What can ai do for me? evaluating machine learning interpretations in cooperative play. In Proceedings of the 24th International Conference on Intelligent User Interfaces (pp. 229-239).

[14] L. B. Fulton, J. Y. Lee, Q. Wang, Z. Yuan, J. Hammer, and A. Perer. 2020, April. Getting playful with explainable ai: Games with a purpose to improve human understanding of ai. In Extended Abstracts of the 2020 CHI Conference on Human Factors in Computing Systems (pp. 1-8).

[15] A. Furnham and H. C. Boo. 2011. A literature review of the anchoring effect. The journal of socio-economics, 40(1), 3542.

[16] C. Garvie 2019. Garbage In, Garbage Out | Face Recognition on Flawed Data. [Online]. Available: https://www.flawedfacedata.com/

[17] D.Y. Geiskkovitch, D. Cormier, S.H. Seo, and J.E Young. 2016. Please continue, we need more data: an exploration of obedience to robots. J. Hum.-Robot Interact. 5, 1 (March 2016), 82-99. DOI: https://doi.org/10.5898/JHRI.5.1.Geiskkovitch

[18] Katy Ilonka Gero, Zahra Ashktorab, Casey Dugan, Qian Pan, James Johnson, Werner Geyer, Maria Ruiz, Sarah Miller, David R. Millen, Murray Campbell, Sadhana Kumaravel, and Wei Zhang. 2020. Mental Models of AI Agents in a Cooperative Game Setting. In Proceedings of the 2020 CHI Conference on Human Factors in Computing Systems (CHI '20). Association for Computing Machinery, New York, NY, USA, 1-12. DOI: https://doi.org/10.1145/3313831.3376316

[19] M. J. Habgood and S. E. Ainsworth. 2011. Motivating children to learn effectively: Exploring the value of intrinsic integration in educational games. The fournal of the Learning Sciences, 20(2), 169-206.

[20] M. J. Habgood, S. E. Ainsworth, and S. Benford. 2005. Endogenous fantasy and learning in digital games. Simulation \& Gaming, 36(4), 483-498.

[21] D. Journet. 2007. Narrative, Action, and Learning: The Stories of Myst. In: Selfe C.L., Hawisher G.E., Van Ittersum D. (eds) Gaming Lives in the Twenty-First Century. Palgrave Macmillan, New York. https://doi.org/10.1057/9780230601765_6

[22] J. Juul. 2010. The game, the player, the world: Looking for a heart of gameness. Plurais Revista Multidisciplinar, 1(2).

[23] R. Kennedy, S. Clifford, T. Burleigh, P. D. Waggoner, R. Jewell, and N. J. Winter. 2020. The shape of and solutions to the MTurk quality crisis. Political Science Research and Methods, 8(4), 614-629.

[24] Y. Kou and X. Gui. 2020. Mediating Community-AI Interaction through Situated Explanation: The Case of AI-Led Moderation. Proceedings of the ACM on Human-Computer Interaction, 4(CSCW2), 1-27.

[25] A. Kühberger. 1998. The influence of framing on risky decisions: A meta-analysis. Organizational Behavior and Human Decision Processes. 75, 1 (1998), 23-55. DOI: https://doi.org/https://doi.org/10.1006/obhd.1998.2781

[26] V. Lai, and C. Tan. 2019. On Human Predictions with Explanations and Predictions of Machine Learning Models: A Case Study on Deception Detection, arXiv preprint arXiv:1811.07901

[27] N. Lane and N. R. Prestopnik 2017, October. Diegetic connectivity: blending work and play with storytelling in serious games. In Proceedings of the Annual Symposium on Computer-Human Interaction in Play (pp. 229-240).

[28] A. C. Madrigal. 2019. How a Feel-Good AI Story Went Wrong in Flint. [Online]. Available: https://www.theatlantic.com/technology/archive/2019/01/how-machine-learning-found-flints-lead-pipes/578692/

[29] P. Madumal, T. Miller, L. Sonenberg, and F. Vetere. 2019. A grounded interaction protocol for explainable artificial intelligence. arXiv preprint arXiv:1903.02409.

[30] T. Miller, P. Howe and L. Sonenberg. 2017. Explainable AI: Beware of inmates running the asylum or: How I learnt to stop worrying and love the social and behavioural sciences. arXiv preprint arXiv:1712.00547.

[31] M. Molineaux, D. Dannenhauer, and D. W. Aha. 2018, January. Towards Explainable NPCs: A Relational Exploration Learning Agent. In AAAI Workshops (pp. 565-569).

[32] K. J. Mullinix, T. J. Leeper, J. N. Druckman, and J. Freese. 2015. The generalizability of survey experiments. Journal of Experimental Political Science, 2(2), 109-138.

[33] M. Narayanan, E. Chen, J. He, B. Kim, S. Gershman, and F. Doshi-Velez. 2018. How do Humans Understand Explanations from Machine Learning Systems? An Evaluation of the Human-Interpretability of Explanation. arXiv preprint arXiv:1802.00682 (2018).

[34] S. Y. Okita, J. Bailenson, and D. L. Schwartz. 2007. The mere belief of social interaction improves learning. In Proceedings of the Annual Meeting of the Cognitive Science Society (Vol. 29, No. 29).

PACM on Human-Computer Interaction, Vol. 5, No. CHI PLAY, Article 228, Publication date: September 2021. 
[35] E. Peer, J. Vosgerau, and A. Acquisti. 2014. Reputation as a sufficient condition for data quality on Amazon Mechanical Turk. Behavior research methods, 46(4), 1023-1031.

[36] M.T. Ribeiro, S. Singh, and C. Guestrin. 2016. "Why Should I Trust You?": Explaining the Predictions of Any Classifier. In Proceedings of the 22nd ACM SIGKDD International Conference on Knowledge Discovery and Data Mining (KDD '16). Association for Computing Machinery, New York, NY, USA, 1135-1144. DOI: https://doi.org/10.1145/2939672.2939778

[37] D. Rumeser and M. Emsley. 2019. Can serious games improve project management decision making under complexity?. Project Management fournal, 50(1), 23-39.

[38] S. Samat and A. Acquisti. 2017. Format vs. content: the impact of risk and presentation on disclosure decisions. In Thirteenth Symposium on Usable Privacy and Security (\{SOUPS\} 2017) (pp. 377-384).

[39] K. Schrier. 2019. Designing Games for Moral Learning and Knowledge Building. Games and Culture. 2019;14(4):306343. doi:10.1177/1555412017711514

[40] C. A. Steinkuehler. 2004. Learning in massively multiplayer online games.

[41] C. Steinkuehler and S. Duncan. 2008. Scientific habits of mind in virtual worlds. fournal of Science Education and Technology, 17(6), 530-543.

[42] K. A. Thomas and S. Clifford. 2017. Validity and Mechanical Turk: An assessment of exclusion methods and interactive experiments. Computers in Human Behavior, 77, 184-197.

[43] Matt Turek. 2019. Explainable Artificial Intelligence (XAI). [Online]. Available: https://www.darpa.mil/program/explainable-artificial-intelligence

[44] A. Tversky and D. Kahneman. 1981. The framing of decisions and the psychology of choice. science, 211(4481), 453458.

[45] J. Villareale and J. Zhu. 2021. Understanding Mental Models of AI through Player-AI Interaction. arXiv preprint arXiv:2103.16168

[46] D. Wang, Q. Yang, A. Abdul, and B. Y. Lim. 2019, May. Designing theory-driven user-centric explainable AI. In Proceedings of the 2019 CHI conference on human factors in computing systems (pp. 1-15).

[47] J. D. Weinberg, J. Freese, and D. McElhattan. 2014. Comparing data characteristics and results of an online factorial survey between a population-based and a crowdsource-recruited sample. Sociological Science, 1.

[48] M. Yin, J.W. Vaughan, and H. Wallach. 2019. Understanding the Effect of Accuracy on Trust in Machine Learning Models. In CHI Conference on Human Factors in Computing Systems Proceedings (CHI 2019), May 4-9, 2019, Glasgow, Scotland.

Received February 2021; revised June 2021; accepted July 2021 